\title{
Are substrate use during exercise and mitochondrial respiratory capacity decreased in arm and leg muscle in type 2 diabetes?
}

\author{
S. Larsen - I. Ara • R. Rabøl • J. L. Andersen • \\ R. Boushel • F. Dela $\cdot$ J. W. Helge
}

Received: 3 December 2008 / Accepted: 10 March 2009/Published online: 25 April 2009

(C) Springer-Verlag 2009

\begin{abstract}
Aim/hypothesis The aim of the study was to investigate mitochondrial function, fibre type distribution and substrate oxidation in arm and leg muscle during exercise in patients with type 2 diabetes and in obese and lean controls.

Methods Indirect calorimetry was used to calculate fat and carbohydrate oxidation during both progressive arm-cranking and leg-cycling exercises. Muscle biopsies from arm and leg were obtained. Fibre type, as well as $\mathrm{O}_{2}$ flux capacity of saponin-permeabilised muscle fibres were measured, the latter by high resolution respirometry, in patients with type 2 diabetes, age- and BMI-matched obese controls, and agematched lean controls.

Results Fat oxidation was similar in the groups during either arm or leg exercise. During leg exercise at higher intensities, but not during arm exercise, carbohydrate oxidation was lower in patients with type 2 diabetes compared with the other groups. In patients with type 2 diabetes, ADP-stimulated state 3 respiration per mg muscle with parallel electron input from complex I+II was lower in $\mathrm{m}$. vastus lateralis compared with
\end{abstract}

S. Larsen $\cdot$ I. Ara $\cdot$ R. Rabøl $\cdot$ R. Boushel $\cdot$ F. Dela $\cdot$

J. W. Helge $(\bowtie)$

Department of Biomedical Sciences,

Center for Healthy Ageing, Faculty of Health Sciences,

Blegdamsvej 32200 N, Denmark

e-mail: jhelge@mfi.ku.dk

I. Ara

Faculty of Health and Sport Sciences, University of Zaragoza,

Huesca, Spain

J. L. Andersen

Institute of Sports Medicine, Bispebjerg Hospital,

Copenhagen, Denmark obese and lean controls, whereas no differences between groups were present in $\mathrm{m}$. deltoideus. A higher percentage of type IIX fibres was seen in $\mathrm{m}$. vastus lateralis in patients with type 2 diabetes compared with obese and lean controls, whereas no difference was found in the deltoid muscle.

Conclusions/interpretation This study demonstrates similar $\mathrm{O}_{2}$ flux capacity, fibre type distribution and carbohydrate oxidation in arm muscle in the groups despite the presence of attenuated values in leg muscle in patients with type 2 diabetes compared with obese and lean controls.

Keywords Exercise - Insulin · Lipid oxidation · Type 2 diabetes

\begin{tabular}{|c|c|}
\hline \multicolumn{2}{|c|}{ Abbreviations } \\
\hline $\mathrm{CS}$ & Citrate synthase \\
\hline FatMax & $\begin{array}{l}\text { Exercise intensity where maximal fat oxidation } \\
\text { occurs }\end{array}$ \\
\hline IPAQ & International Physical Activity Questionnaire \\
\hline LBM & Lean body mass \\
\hline MFO & Maximal fat oxidation \\
\hline mtDNA & Mitochondrial DNA \\
\hline RMR & Resting metabolic rate \\
\hline TG & Triacylglycerol \\
\hline$\dot{V} \mathrm{CO}_{2}$ & Carbon dioxide output \\
\hline$\dot{V} \mathrm{O}_{2}$ & Oxygen uptake \\
\hline$\dot{V} \mathrm{O}_{2 \max }$ & Maximal oxygen uptake \\
\hline
\end{tabular}

\section{Introduction}

Insulin resistance is associated with mitochondrial dysfunction in muscle tissue, but the aetiology of this dysfunction, be it insulin resistance per se, chronic hyperglycaemia or 
accumulation of intracellular lipid, remains unclear $[1,2]$. Moreover it is debated whether the mitochondrial dysfunction observed in type 2 diabetes mellitus is caused by damaged mitochondria [3-5] or simply by a decreased content of mitochondria [6]. There is evidence that lipid accumulation, particularly in muscle and liver, is coupled to insulin resistance [7] and it would therefore seem plausible that mitochondrial dysfunction would be reflected in an attenuated fat oxidation, leading to lipid accumulation. Patients with type 2 diabetes and obese individuals may have a decreased fat oxidation at rest [8,9] as well as during acute exercise at different exercise intensities [10]. However, other studies report similar fat oxidation during exercise in patients with type 2 diabetes and obese individuals [11, 12]. In line with the latter findings one study reported even a lower carbohydrate oxidation in patients with type 2 diabetes compared with matched controls at higher exercise intensities [13], which implies similar or even higher fat oxidation during exercise in patients with type 2 diabetes.

The available data in the literature are mainly derived from leg muscle, but recent studies that reported higher glucose clearance in arm compared with leg muscle in patients with type 2 diabetes [14, 15], together with evidence of heterogeneity in limb fatty acid kinetics in patients with type 2 diabetes compared with controls [16], imply that arm muscle may be somewhat metabolically different from leg muscle. In the above-mentioned studies, fibre type distribution in arm and leg muscle was not measured, and it is therefore possible that muscle phenotypic differences may underlie altered substrate usage. The concept that heterogeneity of fibre type in the musculature of the lower and upper body because of differences in daily physical activity level may account for differences in mitochondrial function and substrate use remains to be explored. Previous studies have implicated type I fibre content in leg muscle as a critical factor in type 2 diabetes [17], but this remain to be demonstrated in arm/upper body muscle [14].

In the present study we investigated whole-body fat and carbohydrate oxidation during arm-cranking or leg-cycling and in resting muscle measured mitochondrial function and fibre type distribution in arm (m. deltoideus) and leg (m. vastus lateralis) muscle in patients with type 2 diabetes and obese and lean controls. Our hypothesis was that substrate oxidation as well as muscle mitochondrial function would be normal in arm muscle compared with healthy controls, but decreased in leg muscle in patients with type 2 diabetes because of a decreased mitochondrial content, indicating that altered substrate usage and mitochondrial function is manifest primarily in locomotor muscle and not in all skeletal muscle in type 2 diabetes mellitus.

\section{Methods}

\section{Participants}

Twenty-three male individuals were recruited and divided into three groups: patients with type 2 diabetes, obese controls matched for age, weight and BMI, and lean controls matched for age. Daily physical activity level was evaluated by the International Physical Activity Questionnaire (IPAQ) [18]. Patients with type 2 diabetes were treated with diet or oral antiglycaemic agents (metformin, sulfonylurea). All participants had normal hepatic and renal function, evaluated by blood screening. None of the controls had a known family history of diabetes or were on treatment for a disease. All participants were fully informed of the nature and the possible risks associated with the study before informed consent was given. The study was approved by the ethical committee of Frederiksberg and Copenhagen municipality (KF 01 320893), and adhered to the Principles of the Declaration of Helsinki.

\section{Experimental protocol}

Participants reported to the laboratory after an overnight fast (10-12 h) at 08.00 hours on three separate days over a 4 week period; each visit was separated by at least 4 days. On the first day, after a $15 \mathrm{~min}$ rest, participants underwent a standard $2 \mathrm{~h}$ OGTT with blood samples taken before and after $2 \mathrm{~h}$ for measurements of plasma glucose concentration (ABL, series 700; Radiometer, Copenhagen, Denmark). This was followed by a whole-body dual-energy X-ray absorptiometry scan (Lunar Prodigy Advance; Lunar, Madison, WI, USA) to determine body composition. An ECG was recorded at rest and during submaximal exercise to exclude individuals with signs of coronary ischaemia. Subsequently an incremental cycling exercise was performed to determine maximal oxygen uptake ( $\dot{V} \mathrm{O}_{2 \max }$ ) (Jaeger ER 800; Würzburg, Germany). On day 2, participants were placed in a supine resting position for 30-45 min, where resting metabolic rate (RMR) was measured by open ventilated hood measurements (Oxycon Pro; Jaeger). After this, a muscle biopsy was obtained from m. deltoideus using a Bergström needle modified for suction [19]. Participants then performed a graded exercise test on a bicycle ergometer to determine maximal fat oxidation (MFO), and the intensity where MFO occurred (FatMax). This test has been described in detail elsewhere [20]. On day 3 , participants again rested in a supine position for 30-45 min and a muscle biopsy was obtained from $\mathrm{m}$. vastus lateralis. Subsequently, participants performed a graded exercise test on an arm-cranking ergometer (Lode, Groningen, the Netherlands) commencing at $20 \mathrm{~W}$ for $5 \mathrm{~min}$ followed by $15 \mathrm{~W}$ increments every $3 \mathrm{~min}$ until the load was 
$65 \mathrm{~W}$. After a 5 min break participants performed a maximal oxygen consumption test with the arms, where the initial work load was $65 \mathrm{~W}$ and then workload increased $15 \mathrm{~W}$ every minute until voluntary exhaustion [21]. The order of the second and third experimental day was randomised, and participants were instructed to eat the same diet the day before each trial. Pulmonary oxygen uptake $\left(\dot{V} \mathrm{O}_{2}\right)$ and carbon dioxide excretion $\left(\dot{V} \mathrm{CO}_{2}\right)$ were measured during exercise using an automated on-line system (Oxycon Pro; Jaeger). Before every test a volume calibration and a calibration of the gas analysers using gases of known composition were performed.

\section{Analytical procedures}

Muscle biopsies The muscle biopsies were divided into three parts: one part was frozen directly in liquid nitrogen within $15 \mathrm{~s}$ after sampling, another part was mounted in a mounting medium (Tissue-Tek; Hounisen Laboratorieudstyr, Risskov, Denmark) and frozen in isopentane cooled by liquid nitrogen. Those parts were stored at $-80^{\circ} \mathrm{C}$ until further analysis. The last part was put in BIOPS (see Kuznetsov et al. [22] for content) and immediately analysed for mitochondrial function.

Citrate synthase (CS) and $\beta$-hydroxy-acyl-CoAdehydrogenase activities were measured as described previously [23]. Muscle glycogen content was analysed by the hexokinase method [24]. Muscle triacylglycerol (TG) content was analysed as described elsewhere [25]. Fibre type, size and capillary density were analysed as described elsewhere [26-28].

Respirometric protocol A detailed description for measuring mitochondrial respiration has been described elsewhere [22]. Minor changes were made and the temperature in the respirometer (Oxygraph-2k; Oroboros, Innsbruck, Austria) during the measurement was $37^{\circ} \mathrm{C}$ (physiological temperature). All studies were carried out after hyperoxygenation to avoid any potential oxygen limitation. All the respiration measurements were made in duplicate performed simultaneously. This method makes it possible to study the mitochondria 'in situ' in the cell.

The protocol used for measuring mitochondrial respiration was: resting respiration (state 2 , no adenylates present) was assessed by addition of malate $(2 \mathrm{mmol} / \mathrm{l})$ a complex I substrate and octanoyl carnitine $(1.5 \mathrm{mmol} / \mathrm{l})$ providing electron input to both complex I and II. State 3 was reached by adding ADP ( $5 \mathrm{mmol} / \mathrm{l})$, adding glutamate $(20 \mathrm{mmol} / \mathrm{l})$, another substrate for complex I. Addition of succinate (10 mmol/l) provided electron input to both complex I and II. Intactness of the outer mitochondrial membrane was established by observing no stimulation of respiration after addition of cytochrome $c(10 \mu \mathrm{mol} / \mathrm{l})$. ATP synthase was inhibited by addition of oligomycin $(2 \mu \mathrm{g} / \mathrm{ml})$, and finally uncoupled respiration was reached by titrating carbonylcyanide-4(trifluoromethoxy)-phenylhydrazone $(0.5 \mu \mathrm{mol} / \mathrm{l})$ in steps until the maximal uncoupled respiration was established. Complex I substrates are generating NADH and complex II substrates are generating $\mathrm{FADH}_{2}$. All chemicals are provided from Sigma-Aldrich (St Louis, MO, USA), Fluka Chemie (Buchs, Switzerland) and Tocris Bioscience (Ellisville, MO, USA).

Blood samples Blood was transferred into tubes containing $0.3 \mathrm{~mol} / \mathrm{l}$ EDTA (for plasma glucose and NEFA measurements) and trasylol/EDTA (for insulin measurements). Tubes were immediately centrifuged at $4^{\circ} \mathrm{C}$ for $10 \mathrm{~min}$ at $2480 \times \mathrm{g}$. The plasma was stored at $-80^{\circ} \mathrm{C}$ until analysis. Insulin in venous plasma was determined using an RIA kit (Insulin RIA100; Pharmacia, Uppsala, Sweden). Plasma glucose was analysed using a conventional commercially available assay on an automatic analyser (Hitachi 612 Automatic Analyzer; Roche, Switzerland). Plasma NEFA concentration was measured using a Wako NEFA-C test kit (Wako Chemical, Neuss, Germany) and determined on an automated analyser (Hitachi 612 Automatic Analyzer). $\mathrm{HbA}_{1 \mathrm{c}}$ was analysed on a Bayer DCA 2000+ (Bayer Healthcare, Elkhart, IN, USA) using a latex immunoagglutination inhibition method.

Calculations Whole-body fat and carbohydrate oxidation was calculated as described previously [29]. For determination of RMR, data obtained during the hood measurements were used; at least $5 \mathrm{~min}$ of the last $15 \mathrm{~min}$ measured was used. The HOMA index was calculated as described by Matthews et al. [30].

Statistics Data are presented as means \pm SE in all figures and tables. For all statistical evaluations, $p<0.05$ was considered significant. One- and two-way ANOVAs with repeated measures for the time factor were performed. Significant main effects or interactions were further analysed by the Holm-Sidak post hoc test. Pearson's correlation analysis was performed to establish the presence of correlations. If the normality test failed, the data were $\log _{10}$ transformed and reanalysed. The statistical analysis was performed using the software program SigmaStat 3.1 (Systat Software, San Jose, CA, USA).

\section{Results}

Age did not differ between the groups (Table 1). In contrast, weight, BMI, lean body mass (LBM) and percent body fat were lower $(p<0.05)$ in lean controls compared with obese controls and patients with type 2 diabetes (Table 1). Blood data are provided in Table 2 and muscle data in Table 3. 
Table 1 Characteristics of the participants

\begin{tabular}{|c|c|c|c|}
\hline Characteristic & $\begin{array}{l}\text { Type } 2 \\
\text { diabetes } \\
(n=8)\end{array}$ & $\begin{array}{l}\text { Obese } \\
\text { controls } \\
(n=8)\end{array}$ & $\begin{array}{l}\text { Lean } \\
\text { controls } \\
(n=7)\end{array}$ \\
\hline Age (years) & $48 \pm 4$ & $37 \pm 2$ & $43 \pm 3$ \\
\hline Weight (kg) & $102 \pm 7$ & $108 \pm 6$ & $82 \pm 4^{\mathrm{a}}$ \\
\hline BMI $\left(\mathrm{kg} / \mathrm{m}^{2}\right)$ & $32 \pm 2$ & $32 \pm 1$ & $25 \pm 1^{\mathrm{a}}$ \\
\hline LBM (kg) & $65 \pm 4$ & $71 \pm 4$ & $61 \pm 2^{\mathrm{b}}$ \\
\hline Body fat $(\%)$ & $33 \pm 2$ & $32 \pm 1$ & $23 \pm 1^{\mathrm{a}}$ \\
\hline Time since diagnosis (years) & $4 \pm 1$ & - & - \\
\hline$\dot{V} \mathrm{O}_{2 \max } \operatorname{leg}\left(\mathrm{ml} \mathrm{kg}^{-1} \mathrm{~min}^{-1}\right)$ & $26 \pm 2$ & $31 \pm 2^{\mathrm{c}, \mathrm{e}}$ & $42 \pm 1^{\mathrm{a}, \mathrm{e}}$ \\
\hline$\dot{V} \mathrm{O}_{2 \max } \operatorname{arm}\left(\mathrm{ml} \mathrm{kg}^{-1} \min ^{-1}\right)$ & $22 \pm 2$ & $22 \pm 1^{\mathrm{e}}$ & $31 \pm 2^{\mathrm{a}, \mathrm{e}}$ \\
\hline Arm vs leg ratio $\left(\dot{V} \mathrm{O}_{2 \max }\right)$ & $0.82 \pm 0.06^{\mathrm{d}}$ & $0.71 \pm 0.03$ & $0.73 \pm 0.05$ \\
\hline $\begin{array}{l}\text { IPAQ energy expenditure } \\
\text { in a normal week }(\mathrm{kJ})\end{array}$ & $641 \pm 148$ & $541 \pm 103$ & $732 \pm 147$ \\
\hline
\end{tabular}

Data are means $\pm \mathrm{SE}$

${ }^{\mathrm{a}} p<0.05$ lean vs obese and diabetes; ${ }^{\mathrm{b}} p<0.05$ lean vs obese; ${ }^{\mathrm{c}} p<0.05$ obese vs diabetes, ${ }^{\mathrm{d}} p<0.05$ diabetes vs lean and obese; ${ }^{\mathrm{e}} p<0.05$ arm vs leg

Whole-body fat oxidation was similar at all intensities during arm or leg exercise in the groups (Fig. 1a,b). MFO was also similar in the groups, and was significantly higher during leg-cycling than during arm-cranking in all groups: patients with type 2 diabetes $(0.32 \pm 0.05$ vs $0.20 \pm 0.03 \mathrm{~g} \mathrm{~min}^{-1}$, respectively), obese $(0.31 \pm 0.04 \mathrm{vs}$ $0.22 \pm 0.02 \mathrm{~g} \mathrm{~min}^{-1}$, respectively), and lean $(0.30 \pm 0.02$ vs $0.18 \pm 0.03 \mathrm{~g} \mathrm{~min}^{-1}$, respectively) controls (Fig. 1a,b). Expressing MFO in $\mathrm{g} \mathrm{min}^{-1}$ ( $\mathrm{kg}$ body weight $)^{-1}$ or $\mathrm{g} \mathrm{min}^{-1}$ $(\mathrm{kg} \mathrm{LBM})^{-1}$ showed similar results (data not shown). The intensity at which MFO occurred (FatMax) during legcycling was similar in patients with type 2 diabetes, obese and lean controls $(46.0 \pm 3.4 \%$ vs $42.4 \pm 2.8 \%$ vs $40.0 \pm 0.8 \%$ $\dot{V} \mathrm{O}_{2 \max }$, respectively), but during arm-cranking FatMax was higher in obese compared with lean controls $(37.9 \pm 1.6 \%$ vs $30.1 \pm 2.0 \% \dot{V} \mathrm{O}_{2 \max }$, respectively), but similar in type 2 diabetes $\left(35.4 \pm 2.2 \% \dot{V} \mathrm{O}_{2 \max }\right)$. Comparison of FatMax from arm-cranking and leg-cycling showed a lower FatMax during arm-cranking in patients with type 2 diabetes and lean controls, while a tendency $(p=0.09)$ towards a lower FatMax during arm-cranking was seen in obese controls. Carbohydrate oxidation was similar in the groups during arm-cranking, but was lower $(p<0.05)$ in patients with type 2 diabetes at intensities above $50 \%$ of $\dot{V} \mathrm{O}_{2 \max }$ compared with the other groups during leg-cycling (Fig. 2a,b).

In $\mathrm{m}$. vastus lateralis, state 3 mitochondrial $\mathrm{O}_{2}$ flux per mg muscle tissue was significantly lower in patients with type 2 diabetes compared with obese controls after adding glutamate. The increase in $\mathrm{O}_{2}$ flux per mg muscle tissue with addition of succinate was lower $(p<0.05)$ in patients with type 2 diabetes compared with obese and lean controls (Fig. 3a,b). No differences in proton leak (state 4 respiration) by adding oligomycin was seen (data not shown). State $3 \mathrm{O}_{2}$ flux per mg muscle tissue was similar in $\mathrm{m}$. deltoideus in all groups for glutamate and succinate (Fig. 3a,b). The addition of succinate resulted in a similar increase in state $3 \mathrm{O}_{2}$ flux in all groups. In patients with type 2 diabetes, state $3 \mathrm{O}_{2}$ flux was similar in $\mathrm{m}$. vastus lateralis and $\mathrm{m}$. deltoideus whereas a higher state $3 \mathrm{O}_{2}$ flux was seen in $\mathrm{m}$. vastus lateralis from obese and lean controls compared with $\mathrm{m}$. deltoideus. No cytochrome $c$ response was observed in $\mathrm{m}$. vastus lateralis or $\mathrm{m}$. deltoideus (data not shown). When $\mathrm{O}_{2}$ flux was normalised for CS activity (index of mitochondrial density) all differences in respiration from $\mathrm{m}$. vastus lateralis between groups disappeared, in contrast to $\mathrm{m}$. deltoideus where lean controls showed a lower $(p<0.05)$ normalised mitochondrial respiration than obese individuals and patients with type 2 diabetes (Fig. 3c,d).

The percentage of type IIX fibres in $\mathrm{m}$. vastus lateralis was higher $(p<0.05)$ in patients with type 2 diabetes compared with obese and lean controls, and patients with type 2 diabetes showed a lower $(p<0.05)$ percentage of type I fibres compared with lean controls (Table 3 ). Fibre type distribution was similar in $\mathrm{m}$. vastus lateralis in obese and lean controls. Fibre type distribution was not different in $\mathrm{m}$. deltoideus between the groups. In patients with type 2 diabetes a higher $(p<0.05)$ percentage of type I fibres and a lower $(p<0.05)$ percentage of type IIA fibres was observed in $\mathrm{m}$. deltoideus compared with $\mathrm{m}$. vastus lateralis, whereas no difference was observed in lean or obese controls. Data on capillarisation are presented in Table 3.
Table 2 Blood and plasma data and calculated HOMA score in the overnight fasted condition in the three groups

Data are means $\pm \mathrm{SE}$

${ }^{\mathrm{a}} p<0.05$ lean vs obese and diabetes; ${ }^{\mathrm{b}} p<0.05$ diabetes vs lean and obese

\begin{tabular}{lccc}
\hline Variable & Diabetes & Obese controls & Lean controls \\
\hline Insulin $(\mathrm{pmol} / \mathrm{l})$ & $95 \pm 18$ & $59 \pm 9$ & $31 \pm 4^{\mathrm{a}}$ \\
Fasting glucose $(\mathrm{mmol} / \mathrm{l})$ & $8.7 \pm 1.4^{\mathrm{b}}$ & $5.3 \pm 0.1$ & $5.3 \pm 0.1$ \\
120 min glucose $(\mathrm{mmol} / \mathrm{l})$ & $13.1 \pm 2.2^{\mathrm{b}}$ & $6.8 \pm 0.6$ & $6.9 \pm 0.4$ \\
$\mathrm{HOMA}$ & $34.7 \pm 6.8^{\mathrm{b}}$ & $13.6 \pm 2.2$ & $7.3 \pm 0.9$ \\
$\mathrm{HbA}_{1 \mathrm{c}}(\%)$ & $7.0 \pm 0.7^{\mathrm{b}}$ & $5.4 \pm 0.1$ & $5.1 \pm 0.1$ \\
$\mathrm{NEFA}(\mu \mathrm{mol} / \mathrm{l})$ & $377 \pm 33$ & $366 \pm 48$ & $345 \pm 54$ \\
\hline
\end{tabular}


Table 3 Fibre type composition, capillarisation, enzyme activity and glycogen and TG content in $\mathrm{m}$. vastus lateralis and $\mathrm{m}$. deltoideus from diabetes patients and obese and lean controls

\begin{tabular}{|c|c|c|c|c|c|c|}
\hline \multirow[t]{2}{*}{ Variable } & \multicolumn{3}{|c|}{ Leg (m. vastus lateralis) } & \multicolumn{3}{|c|}{ Arm (m. deltoideus) } \\
\hline & Diabetes & Obese controls & Lean controls & Diabetes & Obese controls & Lean controls \\
\hline Type I (\%) & $38.5 \pm 5.1^{\mathrm{e}}$ & $47.3 \pm 4.4$ & $55.5 \pm 4.8^{\mathrm{b}}$ & $52.2 \pm 3.2$ & $49.1 \pm 5.0$ & $49.0 \pm 3.4$ \\
\hline Type IIA (\%) & $35.3 \pm 1.5^{\mathrm{e}}$ & $41.1 \pm 4.1$ & $34.2 \pm 4.1$ & $28.1 \pm 2.4$ & $32.8 \pm 2.4$ & $37.5 \pm 4.0$ \\
\hline Type IIX (\%) & $26.2 \pm 4.6^{\mathrm{a}}$ & $11.6 \pm 3.3$ & $10.3 \pm 2.2$ & $19.2 \pm 1.9$ & $18.1 \pm 5.0$ & $13.5 \pm 3.0$ \\
\hline Type I area $\left(\mu \mathrm{m}^{2}\right)$ & $5,171 \pm 468$ & $5,084 \pm 336$ & $4,248 \pm 312$ & $6,054 \pm 714$ & $5,930 \pm 641$ & $4,056 \pm 364^{\mathrm{d}}$ \\
\hline Type II area $\left(\mu \mathrm{m}^{2}\right)$ & $6,227 \pm 831$ & $5,810 \pm 294$ & $4,513 \pm 260$ & $6,923 \pm 985$ & $7,397 \pm 927$ & $5,267 \pm 317$ \\
\hline Capillaries per fibre & $3.91 \pm 0.27$ & $4.45 \pm 0.22$ & $3.87 \pm 0.17^{\mathrm{d}}$ & $4.31 \pm 0.32$ & $4.51 \pm 0.25$ & $4.28 \pm 0.19$ \\
\hline Capillaries per $\mathrm{mm}^{2}$ & $359 \pm 22$ & $420 \pm 31$ & $440 \pm 21^{\mathrm{b}}$ & $359 \pm 25$ & $361 \pm 25$ & $445 \pm 24^{\mathrm{c}}$ \\
\hline CS activity $\left(\mu \mathrm{mol} \mathrm{g}{ }^{-1} \min ^{-1}\right)$ & $66 \pm 8$ & $72 \pm 7^{\mathrm{e}}$ & $93 \pm 7^{\mathrm{c}, \mathrm{e}}$ & $50 \pm 3$ & $46 \pm 8$ & $62 \pm 9$ \\
\hline HAD activity $\left(\mu \mathrm{mol} \mathrm{g}^{-1} \min ^{-1}\right)$ & $82 \pm 8$ & $85 \pm 8$ & $107 \pm 8^{\mathrm{c}}$ & $83 \pm 5$ & $62 \pm 8$ & $85 \pm 11$ \\
\hline Glycogen (nmol/mg) & $329 \pm 32$ & $320 \pm 33$ & $375 \pm 67$ & $302 \pm 25$ & $295 \pm 33$ & $348 \pm 46$ \\
\hline Intramuscular TG (nmol/mg) & $177 \pm 62$ & $233 \pm 49$ & $109 \pm 31$ & $235 \pm 64$ & $207 \pm 37$ & $193 \pm 56$ \\
\hline
\end{tabular}

Data are means \pm SE

${ }^{\mathrm{a}} p<0.05$ diabetes vs lean and obese; ${ }^{\mathrm{b}} p<0.05$ lean vs diabetes; ${ }^{\mathrm{c}} p<0.05$ lean vs obese and diabetes; ${ }^{\mathrm{d}} p<0.05$ lean vs obese; ${ }^{\mathrm{e}} p<0.05$ arm vs leg HAD, $\beta$-hydroxy-acyl-CoA-dehydrogenase

\section{Discussion}

A major finding in the present study is that muscle mitochondrial respiration 'in situ' in permeabilised fibres and carbohydrate oxidation during exercise is attenuated in patients with type 2 diabetes compared with lean and obese controls in leg, but not arm muscle. These observations were accompanied by a fibre type distribution with a lower type I and higher type IIX content in leg, but not arm muscle in patients with type 2 diabetes compared with obese and lean controls. However, surprisingly this did not result in a decreased capacity to oxidise fat as measured by gas exchange during exercise with the upper and lower body in patients with type 2 diabetes compared with lean and obese controls. The findings in the present study support the notion that the effect of diabetes on skeletal muscle mitochondrial function and fibre type expression is exerted on the locomotor muscles of the lower limbs and not the muscle of the arms.

Fat oxidation during exercise was investigated using a progressive incremental exercise test and our data clearly demonstrate no difference in fat oxidation across all exercise intensities between patients with type 2 diabetes, lean and obese controls. This is in direct contrast to data from Ghanassia et al. [10], where a markedly decreased capacity to oxidise fat was observed across all exercise intensities in patients with type 2 diabetes of both sexes compared with controls. Although minor methodological differences are present, where we applied shorter incremental steps of $3 \mathrm{~min}$ and Ghanassia et al. used $5 \mathrm{~min}$, our data are supported by other studies that did not find a difference in fat oxidation during exercise between patients with type 2 diabetes and controls, when measured at one exercise intensity [11, 12, 31, 32]. Achten et al. compared protocols applying both 3 and 5 min intervals and did not observe a difference [20]. Basal plasma NEFA concentrations and intramuscular TG content were not higher in patients with type 2 diabetes compared with lean or obese controls. The present data therefore demonstrate that fat oxidation during aerobic exercise is not attenuated in patients with type 2 diabetes compared with lean and obese controls, despite a lower $\dot{V} \mathrm{O}_{2 \max }$ in patients with type 2 diabetes. It is noteworthy also that, despite lower mitochondrial content and type I fibre content in patients with type 2 diabetes in the present study, fat oxidation during leg exercise was similar in the groups, thus implying that lower mitochondrial content and fat oxidation are not primary factors in the aetiology of type 2 diabetes.

The values for MFO during leg exercise in lean controls are similar to those previously reported in young untrained sedentary controls [29] and the relative exercise intensity where it occurs is also in agreement with other studies [29, 33]. To the best of our knowledge, we are the first to report similar MFO determined during progressive arm exercise in patients with type 2 diabetes, and in obese and lean controls. In a prior study it was demonstrated that fat oxidation during arm-cranking was increased after 42 days of prolonged low-intensity training without changes in $\dot{V} \mathrm{O}_{2 \max }$ [34]. In this study $\dot{V} \mathrm{O}_{2 \max }$ was lower during upperbody exercise in patients with type 2 diabetes and obese 

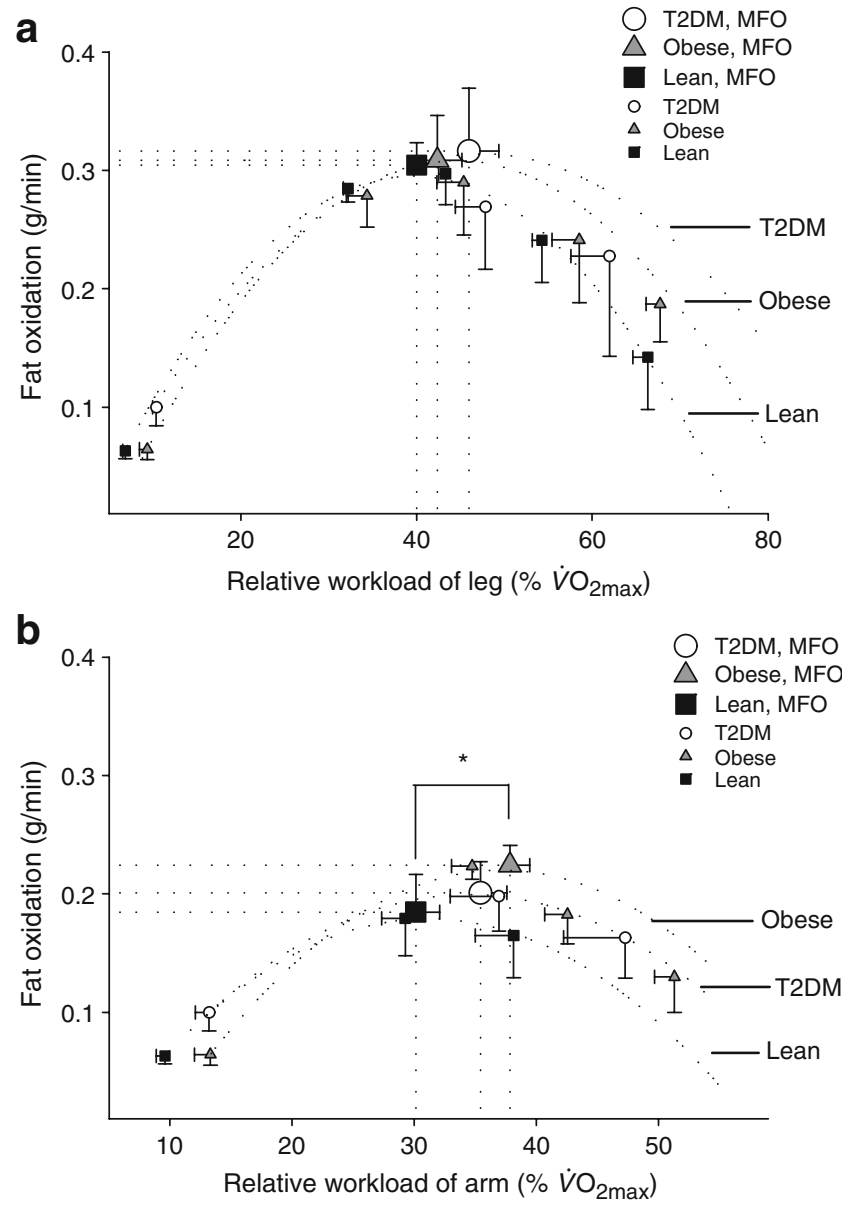

Fig. 1 Fat oxidation during progressive arm or leg exercise calculated by indirect calorimetry during leg exercise (a) or arm exercise (b) in patients with type 2 diabetes and obese and lean controls. Data are means \pm SE. $* p<0.05$ lean vs obese. The large symbols represent FatMax (drop lines). The small symbols are the actual points measured during the test (points are only shown if all the individuals had finished the step). The bell-shaped dotted curves represent the average of the fitted curves for all the participants. T2DM, type 2 diabetes mellitus

control individuals compared with lean controls, but this did not lead to a decreased fat oxidation during upper-body exercise in patients with type 2 diabetes and obese controls.

A prevailing hypothesis in the literature holds that impaired mitochondrial function is a primary cause of type 2 diabetes [4, 6]. Mogensen et al. reported a decreased mitochondrial function in patients with type 2 diabetes compared with matched controls based on experiments performed on isolated muscle mitochondria [4]. In contrast Boushel et al. found a lower mitochondrial respiration per mg muscle in saponin-permeabilised fibres in patients with type 2 diabetes compared with controls but similar muscle mitochondrial function when mitochondrial respiration was normalised to mitochondrial DNA (mtDNA) content per mg muscle or CS activity [6]. The present study corrobo- rates previous findings of a lower mitochondrial respiration in leg muscle in patients with type 2 diabetes, but this pattern is attributed primarily to lower mitochondrial content indicated by a lower CS activity in patients with type 2 diabetes than in lean controls. The lower capillary density per fibre in patients with type 2 diabetes is consistent with a lower mitochondrial content, as these structures are proportionally expressed [35]. The finding that state 3 respiration normalised for CS activity was similar in the legs in the groups when $\mathrm{O}_{2}$ flux was measured with electron flow through complex I+II implies similar qualitative functional characteristics of muscle mitochondria. Taken together, these findings support the concept that mitochondrial function is not impaired per se in the leg muscles of type 2 diabetes patients, but they do have a reduced mitochondrial content. A critical issue is the procedure used to normalise mitochondrial respiration relative to mitochondrial content. In a recent study
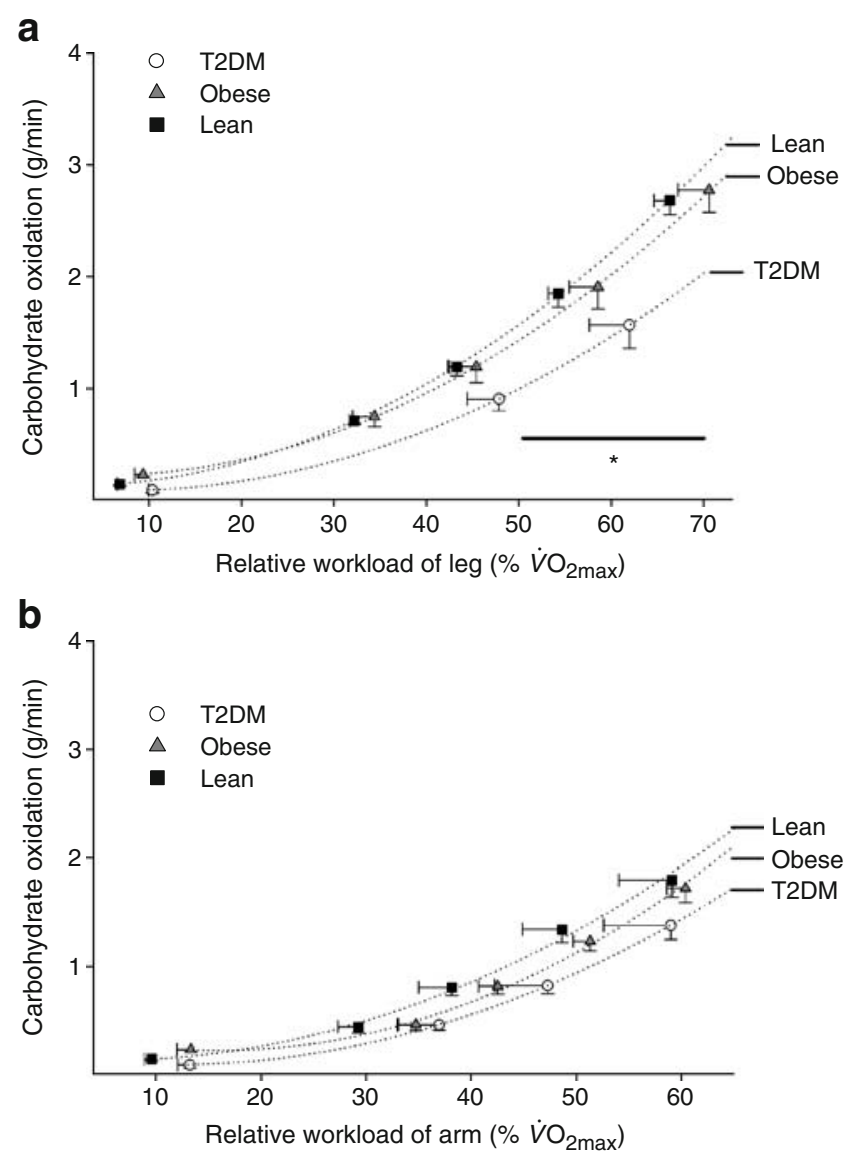

Fig. 2 Carbohydrate oxidation during progressive arm or leg exercise calculated by indirect calorimetry; during leg exercise (a) or arm exercise (b) in patients with type 2 diabetes and obese and lean controls. Data are means \pm SE. ${ }^{*} p<0.05$ diabetes vs lean and obese. The symbols are the actual points measured during the test (points are only shown if all the individuals had finished the step). The dotted curves are the best polynomial fit for the points measured 
a

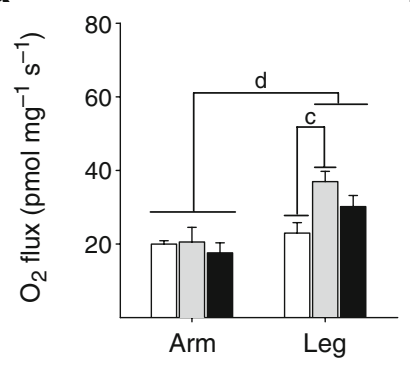

C

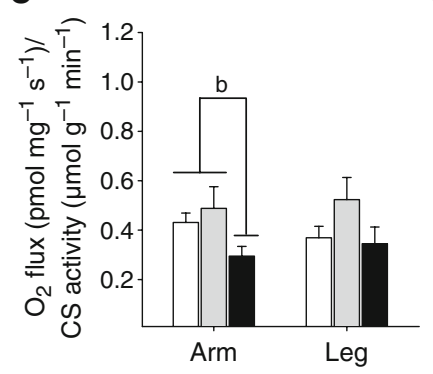

b
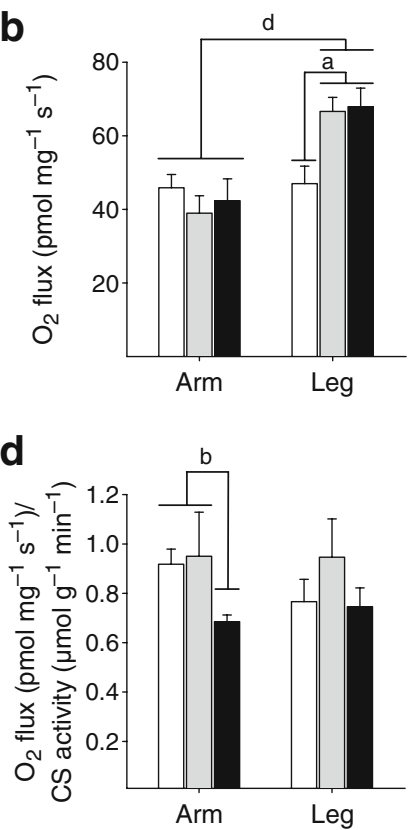

Fig. 3 Mitochondrial respiration $\left(\mathrm{O}_{2}\right.$ flux/mg tissue) in arm (m. deltoideus) and leg ( $\mathrm{m}$. vastus lateralis) muscle from patients with type 2 diabetes (white bars) and obese (grey bars) and lean (black bars) control participants. Mitochondrial respiration was measured with malate, octanoyl carnitine, ADP and glutamate (state 3 respiration with electron input from complex I+II) (a), or malate, octanoyl carnitine, ADP, glutamate and succinate (state 3 maximal coupled respiration with parallel electron input from complex I+II) (b). c Mitochondrial respiration $\left(\mathrm{O}_{2}\right.$ flux per $\mathrm{mg}$ tissue) normalised for mitochondrial content (CS activity) with the same protocol as in panel a. d Mitochondrial respiration $\left(\mathrm{O}_{2}\right.$ flux per mg tissue) normalised for mitochondrial content (CS activity) with the same protocol as in panel $\mathbf{b}$. Data are means \pm SE. ${ }^{a} p<0.05$ diabetes vs lean and obese; ${ }^{b} p<0.05$ lean vs obese and diabetes; ${ }_{p} p<0.05$ obese vs diabetes; ${ }^{\mathrm{d}} p<0.05$ arm vs leg

mitochondrial function was impaired in patients with type 2 diabetes when expressed relative to both mtDNA and CS activity [5], which contrasts with the present results and the findings by Boushel et al. [6], where mitochondrial respiration was normalised to both mtDNA and CS activity. The above discrepancy cannot easily be explained. However, the finding that the arm muscles of patients with type 2 diabetes have completely normal mitochondrial function and expression, whereas the legs show a consistently reduced expression of mitochondria, clearly highlights that the role of mitochondria in the manifestations of patients with type 2 diabetes requires further attention.

Several factors could lead to a decreased mitochondrial content, such as genetic disposition towards a specific fibre type composition and/or an attenuated capacity to tolerate and adapt to physical activity. It has previously been speculated that the upper-body musculature has adapted to be less dependent on muscle usage [14], possibly as a consequence of the upright posture in which only the legs are used for movement. In the present study a higher

percentage of type IIX fibres was found in leg muscle in patients with type 2 diabetes compared with lean and obese controls. However, it was not possible to determine if this was caused by a genetic disposition or because of a lower daily physical activity level or attenuated adaptation to physical activity. In the group with type 2 diabetes patients, five of the eight individuals had a family history of diabetes, but at the same time had similar physical activity levels evaluated by the IPAQ to obese and lean controls, and yet the $\dot{V} \mathrm{O}_{2 \max }$ was lower in patients with type 2 diabetes. The finding of an increased type IIX fibre content in patients with type 2 diabetes has been reported in some $[4,36,37]$ but not all studies [38, 39]. In the present study, fibre type distribution in the arm was similar in all groups and the fibre type distribution in lean controls was in agreement with other previously published values [40-42]. The present findings in arm and leg muscle therefore support the notion that type I fibre content may be a critical component in type 2 diabetes, as previously suggested [14].

In this study a significantly lower carbohydrate oxidation during exercise at the highest intensities was observed in patients with type 2 diabetes compared with the other groups, despite a similar glycogen content found in all the groups. This is supported by the study of Kang et al., which showed a decreased carbohydrate oxidation in patients with type 2 diabetes compared with obese controls at $70 \%$ of $\dot{V} \mathrm{O}_{2 \max }$ [13]. During arm-cranking, carbohydrate oxidation was similar in the three groups, despite the lower upperbody $\dot{V} \mathrm{O}_{2 \max }$ and arm muscle oxidative enzyme activity in patients with type 2 diabetes than in lean controls. Significantly lower values of upper-body $\dot{V} \mathrm{O}_{2 \max }$ and CS activity compared with lower body were seen in obese and lean controls; these values were similar in patients with type 2 diabetes. The values observed in obese and lean controls are similar to those found in normal untrained individuals where $\dot{V} \mathrm{O}_{2 \max }$ obtained during arm-cranking corresponds to approximately $70 \%$ of the value measured during leg-cycling [43]. Interestingly, maximal coupled $\mathrm{O}_{2}$ flux per mg muscle tissue showed a similar pattern, where a higher value was present in obese and lean controls compared with patients with type 2 diabetes in the leg muscle but no difference was present in the arm muscle. Although not significant, the muscle TGs also show this pattern, where stores tend to be lower in lean compared with patients with type 2 diabetes in leg muscle, but not in arm muscle.

A study from our group demonstrated that glucose clearance during a euglycaemic-hyperinsulinaemic clamp was less attenuated in the arm compared with the leg when patients with type 2 diabetes were compared with matched controls [14]. The authors hypothesised that the effect of type 2 diabetes was less marked in the arm compared with the leg and data published later by Reynolds et al. support 
this contention [15]. Overall our data strongly indicate that the muscle and the substrate handling capacity of the upper body in patients with type 2 diabetes is less affected by type 2 diabetes than the leg and lower body when compared with matched lean and obese controls.

In conclusion, our results show that the reduction found in mitochondrial respiration, oxidative capacity and glucose oxidation in patients with type 2 diabetes is only present in muscles of the leg. The attenuated mitochondrial respiration seen in leg musculature from patients with type 2 diabetes are probably caused by the decreased mitochondrial content found. This supports the lack of dysfunction intrinsic to the mitochondria in patients with type 2 diabetes. Whether it is related to the differences in fibre type distribution, or a decreased muscle use, or a diminished responsiveness to physical activity is not known. Interestingly whole-body fat oxidation is not affected by the disease, which could indicate an unknown compensatory mechanism present in the musculature in the lower body from patients with type 2 diabetes.

Acknowledgements We thank J. Bach, T. Beck, J. Isager-Sally and R. Kraunsøe for skilled technical assistance. The study was supported by grants from the Novo Nordisk Foundation, The Foundation of 1870 and the King Christian X Foundation, the Aase \& Ejnar Danielsens Foundation, the Vigo Skovgårds Foundation and the Nordea Foundation.

Duality of interest The authors declare that there is no duality of interest associated with this manuscript.

\section{References}

1. Kelley DE, Goodpaster BH, Storlien L (2002) Muscle triglyceride and insulin resistance. Annu Rev Nutr 22:325-346

2. Kelley DE, Goodpaster BH (2001) Skeletal muscle triglyceride. An aspect of regional adiposity and insulin resistance. Diabetes Care 24:933-941

3. Kelley DE, He J, Menshikova EV, Ritov VB (2002) Dysfunction of mitochondria in human skeletal muscle in type 2 diabetes. Diabetes 51:2944-2950

4. Mogensen M, Sahlin K, Fernstrom M et al (2007) Mitochondrial respiration is decreased in skeletal muscle of patients with type 2 diabetes. Diabetes 56:1592-1599

5. Phielix E, Schrauwen-Hinderling VB, Mensink M et al (2008) Lower intrinsic ADP-stimulated mitochondrial respiration underlies in vivo mitochondrial dysfunction in muscle of male type 2 diabetic patients. Diabetes 57:2943-2949

6. Boushel R, Gnaiger E, Schjerling P, Skovbro M, Kraunsoe R, Dela F (2007) Patients with type 2 diabetes have normal mitochondrial function in skeletal muscle. Diabetologia 50:790796

7. Goodpaster BH, He J, Watkins S, Kelley DE (2001) Skeletal muscle lipid content and insulin resistance: evidence for a paradox in endurance-trained athletes. J Clin Endocrinol Metab 86:57555761

8. Kelley DE, Mandarino LJ (2000) Fuel selection in human skeletal muscle in insulin resistance: a reexamination. Diabetes 49:677683
9. Phielix E, Mensink M (2008) Type 2 diabetes mellitus and skeletal muscle metabolic function. Physiol Behav 94:252-258

10. Ghanassia E, Brun JF, Fedou C, Raynaud E, Mercier J (2006) Substrate oxidation during exercise: type 2 diabetes is associated with a decrease in lipid oxidation and an earlier shift towards carbohydrate utilization. Diabetes Metab 32:604-610

11. Colberg SR, Hagberg JM, McCole SD, Zmuda JM, Thompson PD, Kelley DE (1996) Utilization of glycogen but not plasma glucose is reduced in individuals with NIDDM during mildintensity exercise. J Appl Physiol 81:2027-2033

12. Blaak EE, van Aggel-Leijssen DP, Wagenmakers AJ, Saris WH, van Baak MA (2000) Impaired oxidation of plasma-derived fatty acids in type 2 diabetic subjects during moderate-intensity exercise. Diabetes 49:2102-2107

13. Kang J, Robertson RJ, Hagberg JM et al (1996) Effect of exercise intensity on glucose and insulin metabolism in obese individuals and obese NIDDM patients. Diabetes Care 19:341-349

14. Olsen DB, Sacchetti M, Dela F, Ploug T, Saltin B (2005) Glucose clearance is higher in arm than leg muscle in type 2 diabetes. J Physiol 565:555-562

15. Reynolds TH, Supiano MA, Dengel DR (2007) Regional differences in glucose clearance: effects of insulin and resistance training on arm and leg glucose clearance in older hypertensive individuals. J Appl Physiol 102:985-991

16. Sacchetti M, Olsen DB, Saltin B, van Hall G (2005) Heterogeneity in limb fatty acid kinetics in type 2 diabetes. Diabetologia 48:938-945

17. Gaster M, Staehr P, Beck-Nielsen H, Schroder HD, Handberg A (2001) GLUT4 is reduced in slow muscle fibers of type 2 diabetic patients: is insulin resistance in type 2 diabetes a slow, type 1 fiber disease? Diabetes 50:1324-1329

18. Craig CL, Marshall AL, Sjostrom M et al (2003) International physical activity questionnaire: 12-country reliability and validity. Med Sci Sports Exerc 35:1381-1395

19. Bergstrom J (1975) Percutaneous needle biopsy of skeletal muscle in physiological and clinical research. Scand J Clin Lab Invest 35:609-616

20. Achten J, Gleeson M, Jeukendrup AE (2002) Determination of the exercise intensity that elicits maximal fat oxidation. Med Sci Sports Exerc 34:92-97

21. Smith PM, Doherty M, Price MJ (2006) The effect of crank rate on physiological responses and exercise efficiency using a range of submaximal workloads during arm crank ergometry. Int J Sports Med 27:199-204

22. Kuznetsov AV, Veksler V, Gellerich FN, Saks V, Margreiter R, Kunz WS (2008) Analysis of mitochondrial function in situ in permeabilized muscle fibers, tissues and cells. Nat Protoc 3:965976

23. Andersen JL, Schjerling P, Andersen LL, Dela F (2003) Resistance training and insulin action in humans: effects of detraining. J Physiol 551:1049-1058

24. Karlsson J, Diamant B, Saltin B (1970) Muscle metabolites during submaximal and maximal exercise in man. Scand J Clin Lab Invest 26:385-394

25. Helge JW, Biba TO, Galbo H, Gaster M, Donsmark M (2006) Muscle triacylglycerol and hormone-sensitive lipase activity in untrained and trained human muscles. Eur J Appl Physiol 97:566572

26. Brooke MH, Kaiser KK (1970) Three 'myosin adenosine triphosphatase' systems: the nature of their $\mathrm{pH}$ lability and sulfhydryl dependence. J Histochem Cytochem 18:670-672

27. Qu Z, Andersen JL, Zhou S (1997) Visualisation of capillaries in human skeletal muscle. Histochem Cell Biol 107:169-174

28. Andersen JL, Aagaard P (2000) Myosin heavy chain IIX overshoot in human skeletal muscle. Muscle Nerve 23:10951104 
29. Nordby P, Saltin B, Helge JW (2006) Whole-body fat oxidation determined by graded exercise and indirect calorimetry: a role for muscle oxidative capacity? Scand J Med Sci Sports 16:209-214

30. Matthews DR, Hosker JP, Rudenski AS, Naylor BA, Treacher DF, Turner RC (1985) Homeostasis model assessment: insulin resistance and beta-cell function from fasting plasma glucose and insulin concentrations in man. Diabetologia 28:412-419

31. Boon H, Blaak EE, Saris WH, Keizer HA, Wagenmakers AJ, van Loon LJ (2007) Substrate source utilisation in long-term diagnosed type 2 diabetes patients at rest, and during exercise and subsequent recovery. Diabetologia 50:103-112

32. Borghouts LB, Wagenmakers AJ, Goyens PL, Keizer HA (2002) Substrate utilization in non-obese Type II diabetic patients at rest and during exercise. Clin Sci (Lond) 103:559-566

33. Venables MC, Achten J, Jeukendrup AE (2005) Determinants of fat oxidation during exercise in healthy men and women: a crosssectional study. J Appl Physiol 98:160-167

34. Helge JW, Lundby C, Christensen DL et al (2003) Skiing across the Greenland icecap: divergent effects on limb muscle adaptations and substrate oxidation. J Exp Biol 206:1075-1083

35. Weibel ER, Taylor CR, Gehr P, Hoppeler H, Mathieu O, Maloiy GM (1981) Design of the mammalian respiratory system. IX. Functional and structural limits for oxygen flow. Respir Physiol 44:151-164
36. Oberbach A, Bossenz Y, Lehmann S et al (2006) Altered fiber distribution and fiber-specific glycolytic and oxidative enzyme activity in skeletal muscle of patients with type 2 diabetes. Diabetes Care 29:895-900

37. Hickey MS, Carey JO, Azevedo JL et al (1995) Skeletal muscle fiber composition is related to adiposity and in vitro glucose transport rate in humans. Am J Physiol 268:E453-E457

38. He J, Watkins S, Kelley DE (2001) Skeletal muscle lipid content and oxidative enzyme activity in relation to muscle fiber type in type 2 diabetes and obesity. Diabetes 50:817-823

39. Kelley DE, Simoneau JA (1994) Impaired free fatty acid utilization by skeletal muscle in non-insulin-dependent diabetes mellitus. J Clin Invest 94:2349-2356

40. Helge JW, Overgaard K, Damsgaard R et al (2006) Repeated prolonged whole-body low-intensity exercise: effects on insulin sensitivity and limb muscle adaptations. Metabolism 55:217-223

41. Mavidis A, Vamvakoudis E, Metaxas T et al (2007) Morphology of the deltoid muscles in elite tennis players. J Sports Sci 25:1501-1506

42. Desplanches D, Hoppeler H, Mayet MH, Denis C, Claassen H, Ferretti G (1998) Effects of bedrest on deltoideus muscle morphology and enzymes. Acta Physiol Scand 162:135-140

43. Secher NH, Ruberg-Larsen N, Binkhorst RA, Bonde-Petersen F (1974) Maximal oxygen uptake during arm cranking and combined arm plus leg exercise. J Appl Physiol 36:515-518 